\title{
Effects of erring behavior in a human-robot joint musical task on adopting Intentional Stance toward the iCub robot.
}

\author{
Ciardo, F., De Tommaso, D., and Wykowska, A., Member, IEEE
}

\begin{abstract}
In this study, we examined whether the likelihood of attributing intentionality to robots is influenced by the human-likeness of errors during HRI. To this end, we designed an experimental protocol in which users performed a melody in a joint task with the $\mathrm{iCub}$ robot. We programmed the $\mathrm{iCub}$ robot to make an error in $60 \%$ of the repetitions. For half of the users, in the erroneous trials, the robot displayed a human-like error, i.e. switched one element of the melody by pressing the wrong key. For the other half of users, the robot erred mechanically, i.e., it interrupted to play the melody and moved back and forth between two keys in an "endless" loop. Before and after the joint musical task, we administered the InStance Test to evaluate the likelihood of treating the robot as an intentional agent. Results showed that mechanical errors during HRI reduced intentionality attribution toward the robot.
\end{abstract}

\section{INTRODUCTION}

In the recent 20 years, we have witnessed a surge in the presence of artificial agents in our environments [1]. The scientific effort of the Human-Robot Interaction (HRI) community focused on the development of AI agents able to detect, classify, and reproduce human behavior $[2,3]$ However, sociality is more than just mimicking, and underlying human interactions is the ability to extend the temporal horizon of our action planning and to act in anticipation of others' actions rather than simply responding to them [4]. More specifically, humans sueceed in social interactions, thanks to a complex plethora of pechanisms [5] that, on the one side, allows us to understand others' internal states and, on the other side, to anticipate their actions. Indeed, expectations about others' behavior are crucial to anticipate co-agents' errors during a joint task and to prevent his/her errors, allowing one to syeessfuly "stay in the loop". However, as artificial agents increasingly populate our environments, we are becoming used to interacting with various types of artif(cial systems, like vocal assistants and robots. In the gase of interactions with artificial agents, expectations and pred ctions about their behavior might rely on different strategies compared to those applied to other humans especially when related to erring behavior. According to Daniel Dennett $[6,7]$, to explain and predict other humans' behavior we adopt an Intentional Stance, namely, we explain and predict their behavior as resulting from mental operations. Thus, when we face a human coagent making an error, we can recognize which operations failed. For example, if your partner forgets the pin code of the bank account, most probably you will explain the error as a failure in recalling from memory the correct number sequence. However, when facing artificial and mechanical

F. C. is with the Italian Institute of Technology, Genova, 16152 Italy (Francesca.Ciardo@iit.it).

D.D.T. is with the Italian Institute of Technology, Genova, 16152 Italy (Davide.DeTommaso@iit.it). systems, according to Dennett, humans explain and predict their behavior (and failures) referring to the way they yere designed or programmed to act, i.e. they adopt the Design/Mechanical Stance. For exanple, if while you are writing a manuscript your computer suddenly shuts down because of a low battery, you wopd interpret $x$ as the consequence of the fact your laptop is not designed to work without electricity. When the co-agent is/a humanoid robot, things are less clear. Indeed, humanoid robots belong to a particular category of artifficial agents [8] that have been intentionally designed to esemble the human appearance and interact with humans in theiv natural environment $[9,10]$. These two characteristics of humanoid robots can potentially trigger the adoption of the intentional stance during an HRI scenario [for a review see 11]. On the other hand, as manmade artifacts, they call for adopting the design stance. Indeed, a recent series of studies showed that humans can adopt eithe the intentional or the design/mechanical stance [1, 12] to explain behaviors of the iCub robot [13] For xample, Marchesi and colleagues $[12,14]$ asked to users to jugge between two different descriptions which one was better to explain the behaviors of the iCub robot or a human agent displayed in a visual scenario. The two possible explanations either referred to intentional or design stances. Results showed that at the explicit level, people chose more often the mentalistic descriptions for the human agent and the mechanistic descriptions for the robot. However, such a pattern was not reflected at the implicit level in response times associated with the robotic agent, suggesting that at the implicit level, both stances are processed as "equally likely" to explain the behavior of a humanoid robot.

Interestingly, individual differences play a crucial role in adopting Intentional Stance towards humanoid robots. For, example, Bossi et al [15] provided evidence that individual biases toward treating robots as either intentional agents or mechanistic artifacts can be detected at the neural level, already in a resting state EEG signal, before any interaction with the robot. Specifically, the authors found that EEG beta activity during resting state was predictive of whether people were inclined toward interpreting iCub's behavior as either mechanistic or intentional. Adopting the intentional or design stance toward a robot is a crucial topic in HRI since the two stances imply not only two different ways of interpreting its behavior but, most importantly, the two stances elicit different expectations about how it should behave. This is particularly relevant in joint action scenarios where the behavior of the robot violates our expectations, like when an error or a failure occurs.

A. W. is with the Italian Institute of Technology, Genova, 16152 Italy (Agnieszka.Wykowska@iit.it). 
A possible explanation for why people could adopt the Intentional Stance towards artificial agents could be that when they do not have a rich and well-grounded knowledge of the system they are facing, as in the case of robots, the perceived similarity to humans plays a critical role. Thus, they tend to adopt the Intentional Stance towards robots with higher physical or behavioral similarity to humans [16, 17]. Consequently, as people gain more expertise regarding robots the less likely they should be to adopt the Intentional Stance. In the present study, we tested this hypothesis by focusing on the behavioral similarity between the iCub and a human. Specifically, we manipulated the human-likeness of erring behavior.

Errors and failures are common events in everyday joint actions. Indeed, in a joint task, our co-agent can violate our expectations by committing an error, despite having learned correctly his/her part of the task or being well-trained. This can apply to both natural and artificial co-agents. However, what differs between natural and artificial agents are the consequences of those failures on how we perceive and interpret the agent. Indeed, when the co-agent is another human being, his/her errors in a joint task do not affect the adoption of Intentional Stance toward him/her, as we would still interpreter his/her behavior as referring to mental states. When the agent is a robot, however, the way its behavior violates our expectations, in a human-like or mechanical way, may affect differently the likelihood of adopting Intentional Stance toward it.

\section{AIM}

The present study aimed to investigate whether the likelihood of adopting Intentional Stance towards robots is influenced by the type of error (human-like vs. mechanistic) that the robot displays during a joint task. To this end, we designed an experimental protocol in which users performed a melody in a joint task with the iCub robot. We programmed the iCub robot to make an error in $60 \%$ of the repetitions. For half of the users, in the erroneous trials, the robot displayed a Humanlike error, i.e. switched one element of the melody by pressing the wrong key. For the other half of users, the robot erred mechanically, i.e., it interrupted to play the melody and moved back and forth between two keys in an "endless" loop. In the present study, the two types of erring behavior set a boundary between the user and the robot, since the mechanical error cannot be considered a feature of human agents, whereas a mismatch in sequence execution resembles a memory failure that often occurs in humans. We examined the adoption of Intentional Stance towards robots before and after a joint musical task, employing the Intentional Stance (InStance) Test developed and validated from our lab [8, 12, $14,15,18,19]$. Also, before performing the task, we evaluated individual differences in attitudes towards robots to test whether they predicted the variation in the likelihood of adopting Intentional Stance before and after the musical joint task.

\section{MATERIALS AND METHODS}

\section{A. Sample}

Thirty-seven right-handed adults (mean age: $22.4 \pm 2.9,13$ Males) took part in this study. All users had a normal orcorrected-to-normal vision and were naïve to the purpose of the study. The study was conducted under the ethical standards laid down in the 2013 Declaration of Helsinki and approved by the local ethical committee (Comitato Etico Regione Liguria). All users gave written informed consent before the experiment.

\section{B. Apparatus and Stimuli}

The experimental setup was a multi-modal human-robot interactive scenario, where the user and the robot interacted with a touch screen by producing a set of periodic audio sequences. The apparatus consisted of a controlling PC, a multi-touch screen $(1099.4 \times 634.0 \times 36.8 \mathrm{~mm}, 60 \mathrm{~Hz})$, and an $\mathrm{iCub}$ humanoid robot [13]. The experiment was designed and implemented using the Opensesame program, a Python-based experiment builder [20]. The experiment, running on the controlling PC, consisted of a) a front-end module based on a custom GUI for allowing the user and the robot to interactively play on a circular music pad; and b) a back-end module programmed for controlling the robot actions and for collecting responses of the users (taps on the screen).

The actions of the robot involved its right arm by reproducing a tapping behavior on the screen for playing the different notes. These actions were composed by using six right arm poses (one for each key of the music pad) and an additional one as starting/end pose for each repetition. The arm trajectories were generated at runtime during the experiment from one target location to another. To achieve this behatior we controlled iCub's arm with the YARP IPositionControl, a position controller implemented following a minimum jerk profile [21]. iCub's pointing gesture was pre- efined os in [22]. The requested trajectory time for moving the ann from one location to another was programmed to the same for all the transitions, namely $350 \mathrm{~ms}$. Users performed the task standing stand next to iCub and facing the fouch screen (see Fig. 1). They were presented with a black screen divided into two equal portions by a white Midline. In each hemifield of the screen, a music pad waspresented. The pad consisted of six colored dots (Ø: 5 cro) poditioned on the vertices of a hexagon, all equidistant from the center (see Fig. 1). Each dot corresponded to a peciffc tone. The pad was centered on iCub's right arm tone duration was $450 \mathrm{~ms}$ and it was estimated en pirically based on iCub's minimum period achievable in the sequence. Users performed the tapping sequence-always with their right arm.

C. Procedure

Attitudes towards robots were evaluated at the beginning of the experimental session, by administering the following questionnaires:

- The Frankenstein Syndrome Questionnaire (FSQ), a selfreport scale that investigates the anxiety perceived towards robots in contexts of interaction [23];

- The Negative Attitudes towards Robots Scale (NARS), a self-report scale that investigates negative attitudes towards robots [24]; 
- The Robotic Social Attitude Scale (RoSAS), a self-report questionnaire that investigates the attribution of anthropomorphic characteristics to robots [25].

Questionnaires administration and data collection were controlled by Opensesame software. [20].

The InStance test was administered immediately before and after taking part in the interactive task with the robot. It consists of 34 items in which the iCub robot is depicted in a scenario composed of 3 images while it is performing a task alone or with human agents. Each scenario is presented with two possible explanations, one describing iCub's behavior referring to mental states and using a mentalistic vocabulary (i.e., to want or to desire). The second one, explaining the behavior of the robot in mechanical terms using a technical vocabulary (i.e., to scan or to calibrate). Users are asked to decide which description fits better the scenario and to move a slider to the preferred description. On each extreme of a range within which a slider can move, there is a mentalistic or mechanistic statement, see Fig. 2. The Instance Test Score (ISS) ranges from 0 to 100 , being 0 the most mechanistic and 100 the most mentalistic. Users were administered two different versions of the test (pre-and post-interaction), each composed of 17 items randomly selected items.

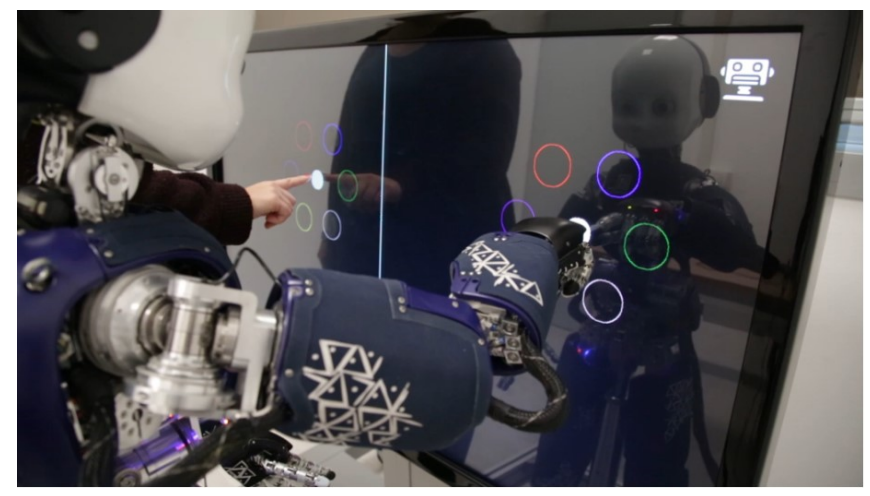

Fig. 1 Interactive task set up

Finally, users were asked to stand next to the robot and perform a joint musical task. Firstly, they had to create their melody sequence. The melody sequence was composed of a sequence of six different tones repeated four times. Then they were asked to teach their melody to iCub. The robot was programmed to follow users' tapping with a sequentially decreasing delay. The delay represents the iCub's response time for tapping a single dot to the human's tap, that is, the time between the detection of the user's tap and iCub's tap. The values have been selected empirically taking into account iCub's arm movement trajectory time $(350 \mathrm{~ms}) \pm$ estimated variability of the position controller (100ms). In the beginning, the robot performed the melody with a delay of $650 \mathrm{~ms}$ with respect to the user performance ( 6 repetitions). Then the delay was reduced to $550 \mathrm{~ms}$ (6 repetitions) and finally reduced to $450 \mathrm{~ms}$ (10 repetitions). Reducing sequentially the delay of iCub's tap among repetitions allowed us to induce the belief that the robot was gradually learning the correct sequence played by the user.

Finally, users were asked to perform a duet with the iCub robot. They were explicitly instructed to coordinate their performance with the iCub robot to keep synchrony and do not make errors in playing the melody. The iCub was programmed to play following the average period estimated from the last four repetitions of the teaching phase. Specifically, the intertap interval between two consecutive taps was equal to the average time differences between two consecutive taps collected from the human users in the last four repetitions of the teaching phase. In this way, we ensured that the robot was playing according to a tempo tailored to each user. The duet task consisted of 22 repetitions of the same melody sequence. In $60 \%$ of the repetitions, we programmed the robot to make an error in playing the sequence. For half of the users, the iCub switched one element of the melody by pressing the wrong key (Human-like Erring Behavior), while for the other half of users it interrupted the melody and moved back and forth between two keys in an 'endless' loop (Mechanical Erring Behavior), see Video 1.
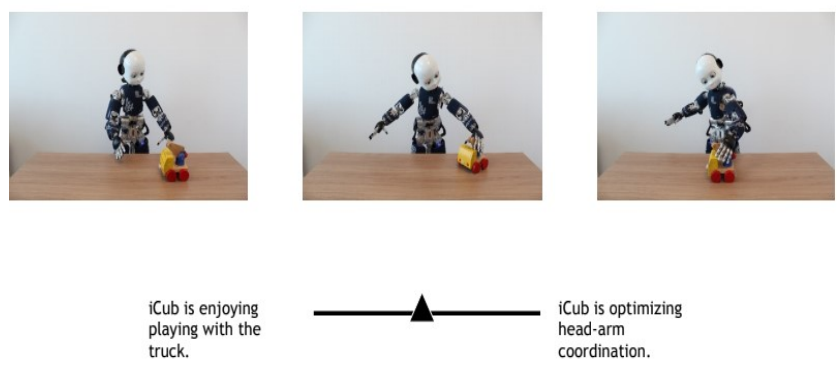

Fig. 2 Example of InStance Test items

\section{DATA ANALYSIS}

The data of five users have been excluded due to a technical failure of the program or the robot during the interactive task. Therefore, data analysis was conducted on a sample size of $\mathrm{N}=32$. Average Instance scores (ISS) were submitted to a repeated-measures analysis of variance (ANOVA) with Session (Pre vs. Post) as within-subject factor and Erring Behavior Condition (Human-like vs. Mechanical) as a between-subject factor. When necessary, comparisons were performed using paired-samples t-tests

For each participant, we estimated the difference between the ISS after interacting with the robot and before, i.e. $\Delta$ ISS. Thus, $\Delta \mathrm{ISS}<0$ indicates that $\mathrm{ASS}$ score reduced in the postsession compared to the ISSreported before interacting with the robot. Whereas, $\triangle \mathrm{IS}>0$ wouldyindicate that the ISS increased after the interaction with the robot. A decrease in ISS denotes a more mechanistic stance while increasing a more mentalist

The $\triangle$ ISS vas modeled using linear general models for FSQ, NARS, and RoSAS subscale, with Erring Behavior Condition as a covariate. Analyses were conducted using JASP and R-studio[26].

\section{RESUlts}

Results showed a significant Erring behavior Condition* Session interaction $\left[\mathrm{F}_{1,30}=4.55, \mathrm{p}=0.041, \mathrm{n}_{\mathrm{p}}{ }^{2}=.13\right]$. Planned comparisons showed that users who interacted with the iCub robot displaying a mechanical error reported a lower ISS in the post-interaction session compared to the pre-interaction session $\left[43.49\right.$ vs. 39.10, respectively; $\mathrm{t}_{30}=-2.80, \mathrm{p}=.013$, 
$\left.\mathrm{d}^{\prime}=.70\right]$. Whereas no significant differences in the ISS before and after the interactive task were found for those users who interacted with the human-like erring robot [39.09 vs. 40.38 , respectively, $t_{30}<1$ ], see Fig 3 . No other main effect was significant [all $\mathrm{p}>0.25$ ].

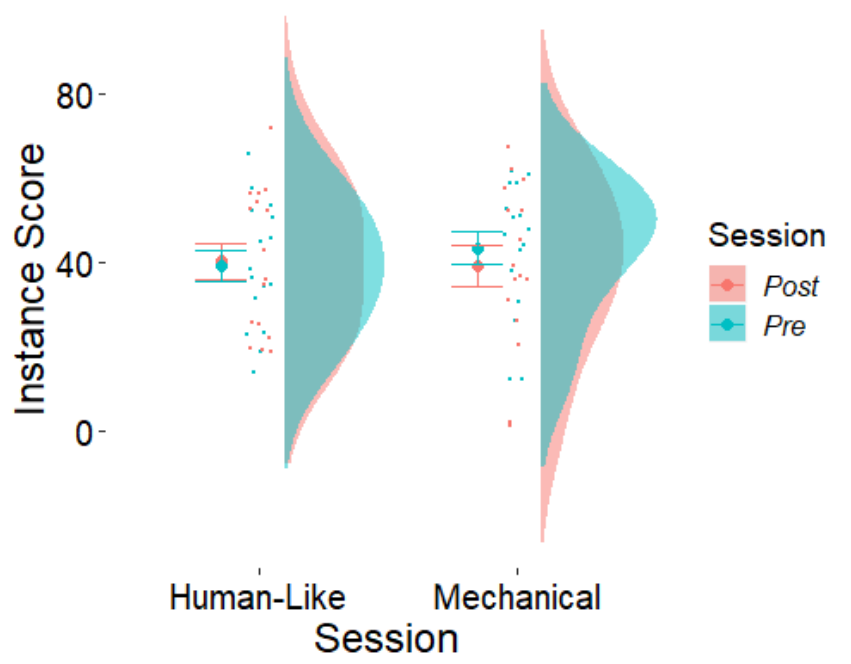

Fig. 3 Mean InStance Score plotted as a function of Erring Behavior Condition (Human-like vs. Mechanical) and Session (Pre vs. Post). Error bars represent standard errors.

The analysis on the effect of individual differences on the variation of the ISS before and after and interactive task revealed that the $\triangle \mathrm{ISS}$ was predicted by the "Warmth" subscale of the RoSAS $\left[\beta=-4.67, \mathrm{t}_{28}=-3.08, \mathrm{p}=0.005\right]$.

Specifically, higher scores on the Warmth subscale predicted smaller or negative variations (i.e. $\Delta \mathrm{ISS} \leq 0$ ) in the ISS score across the two sessions. However, this was true only for users who interacted with the robot depicting a human-like behavior $\left[\beta=-4.67, \mathrm{t}_{28}=-2.48, \mathrm{p}=0.029\right]$. No significant effect was found for the mechanical erring behavior group $\left[\beta=0.17, \mathrm{t}_{28}<1\right]$, see Fig. 4 .

The analysis revealed also a marginal effect of negative emotions in interaction with robots on the $\Delta$ ISS $[\beta=-2.03$, $\left.\mathrm{t}_{28}=2.02, \mathrm{p}=0.054\right]$. Such a pattern was driven by the Human-like Erring behavior condition, $\left[\beta=2.57, \mathrm{t}_{28}=1.92\right.$, $\mathrm{p}=0.066]$. However, given the lack of significance, we did not addressed this interaction further, see Fig.5.

\section{DISCUSSION}

The present study aimed to investigate whether the likehood of adopting Intentional Stance towards robots is influenced by the type of erring behavior of the robot in a joint task. To this end, we designed an experimental protocol in which users performed a musical joint task with the iCub robot. We programmed the iCub robot to make an error in $60 \%$ of the repetitions. For half of the users, in the erroneous trials, the robot displayed a human-like error, whereas, for the other half of users, the robot erred mechanically. We addressed the likelihood of adopting Intentional Stance before and after the joint musical task using the InStance Test. We hypothesized that behavioral similarity between $\mathrm{iCub}$ and human behavior during the interactive task should affect the likelihood to adopt the Intentional Stance.

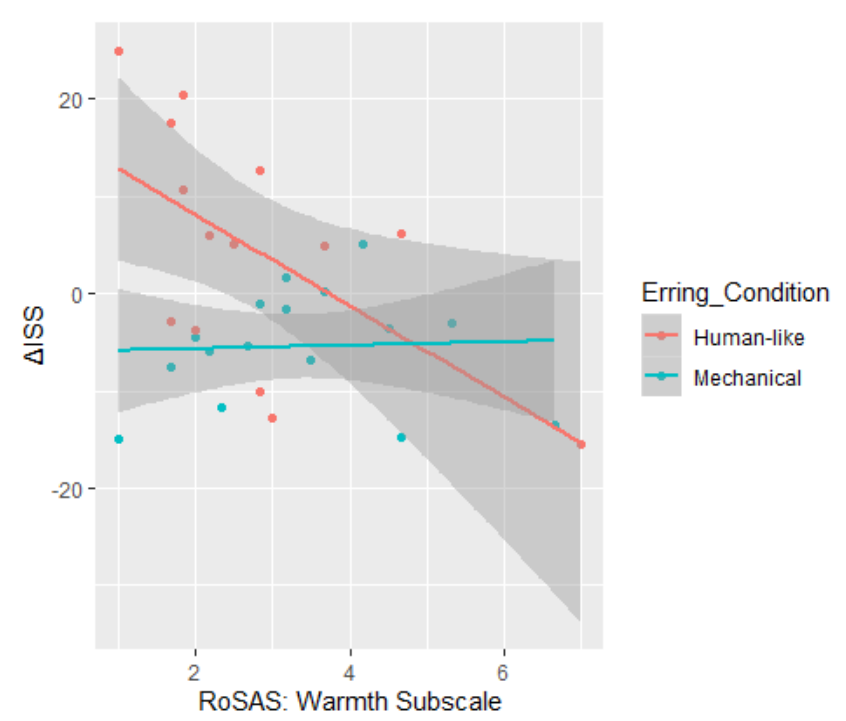

Fig. 4 Mean $\triangle I S S$ plotted as a function of Erring Behavior Condition (Human-like vs. Mechanical) and score on the Warmth subscale of the RoSAS.

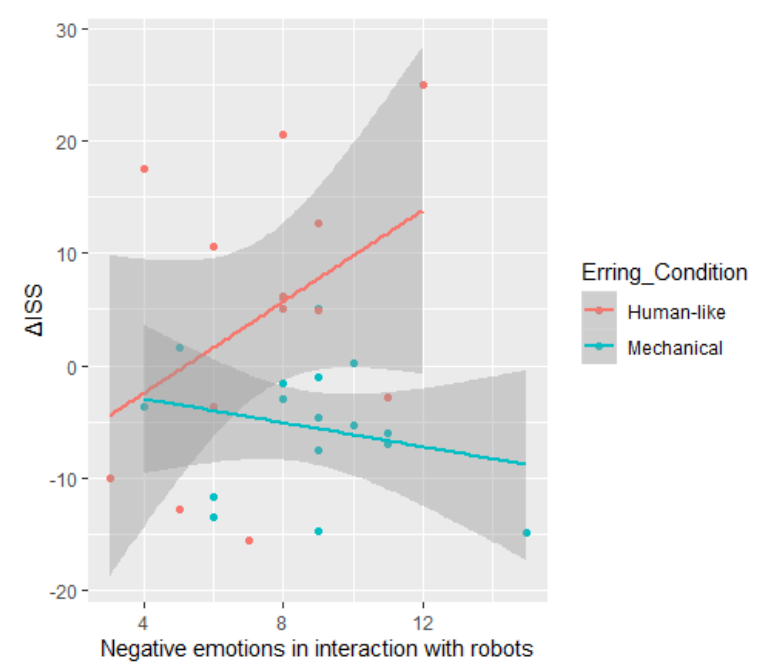

Fig. 5 Mean $\Delta$ ISS plotted as a function of Fring Beharior Condition (Human-like vs. Mechanical) and scores for Negative Emotions in Interaction wi

Results showed that the ISS score was reduced after the interactive task only for hose ysers yto interacted with the $\mathrm{iCub}$ robot displaying a mechanical error. Users who interacted with the iCub fobot producing human-like errors showed a stableuss aeross the two sessions. Such a pattern suggests that the int raction with the human-like erring robot did not change the attitudes toward it, and users continued to consider both stances as "equally likely" to explain the behavio of jeub. Such ambiguity was eliminated for those users tho were exposed to mechanical errors. In this latter case, interacting with a robot that commits an error outside the human repertoire brought users to adopt more often the Design/Mechanical Stance.

Moreover, our results indicate that individual differences in a priori attitudes towards robots predict the variation in the ISS between the pre-and the post- sessions. Specifically, results indicate that individual differences in perceiving robots as "Warm" predicted the $\Delta$ ISS. 
Our results are in line with previous works investigating the violation of expectations in non-verbal communication during HRI [27, 28], showing that when people experience unexpected behavior in HRI, the value (positive or negative) of the violation affects the possibility to perceive the robot as a social partner. According to the expectancy violations theory [29], when co-agents violate our expectations, we react differently depending on the value (positive or negative) of the violation. This is also mediated by individual differences in the pre-interaction state $[29,30]$. When the robot positively violated users' expectations, i.e. improving their performance, they tended to accept more the robot as an interaction partner. Conversely, violations of expectations that negatively affected users' performance resulted in negative feelings and a lower acceptance of it.

Our results extend this previous evidence by showing that the human-likeness of the behavior through which the robot violates users' expectations influence not only social acceptance of the robot but also the perception of it as an intentional agent. Specifically, human-like erring behavior appears to be crucial to sustaining intentionality attribution even when the interaction with the robot is unsuccessful. Interestingly, our results also showed that individual differences in attitudes toward robots predicted the variation in the likelihood of adopting Intentional Stance only when the $\mathrm{iCub}$ behaved in a human-like way, suggesting that the more negative those attitudes are before the interaction, the higher is the likelihood to adopt Intentional after the interaction with a robot resembling human erring behavior.

To sum up, our results suggest that human-likeness of errors during HRI tasks is crucial to ensure that users continue treating robots as intentional agents, even following the occurrence of a failure.

Our results are particularly relevant to the discussion about the Out of the Loop (OOTL) phenomenon, i.e., a decrease in human performance in taking control of the situation when an automatized system failure occurs, due to over-confidence in automation. Humans that are OOTL usually take longer or are unable to detect the failure or to decide if and how they should intervene $[31,32]$. The OOTL phenomenon has been listed as one of the major causes of incidents in a highly automatized work environment, such as air traffic control in civilian aviation [32]. The main problem in coordination with artificial agents may not be the complexity of the system itself, but rather the inappropriate design of the interaction with a human being [33]. Specifically, it has been proposed that when an artificial agent's failure occurs, users end up being OOTL because they are not able to represent artificial agents' intentions and to predict the sequence of events that will occur. Our results suggest that programming the robot to resemble human behavior even when it fails can sustain intentionality attribution, thus it might help users to better react and recover the robot's failure, to successfully reach the task goal in HRI.

Future HRI studies should address this hypothesis by focusing on how the human-likeness of erring behavior of the robot can help users to maintain their performance accurately and successfully stay in the loop in HRI.

\section{CONCLUSIONS}

To conclude, our study showed that attribution of intentionality to a humanoid robot can be modulated by the way it commits errors in a joint task. If the way of erring appears mechanical to users, they are more likely to reduce the likelihood of treating the robot as an intentional agent. This result is of interest not only for fundamental research in HRI but also for the applied field of human factors, where factors that reduce out-of-the-loop phenomenon need to be considered.

\section{ACKNOWLEDGMENT}

This work has received support from the European Research Council under the European Union's Horizon 2020 research and innovation program, ERC Starting Grant, G.A. number: ERC - 2016-StG-715058, awarded to Agnieszka Wykowska. The content of this paper is the sole responsibility of the authors. The European Commission or its services cannot be held responsible for any use that may be made of the information it contains.

\section{REFERENCES}

[1] P. Stone, R. Brooks, E. Brynjolfsson, R. Calo, O. Etzioni, G. Hager, ... A. Teller. Artificial intelligence and life in 2030: One hundred year study on artificial intelligence: Report of the 2015-2016 study panel. Stanford: CA. (2016).

[2] R. Lai W. Lin, \& Y. Wu. Review of research on the key technologies, application fields and development trends of intelligent robots. In International conference on intelligent robotics and applications, pp. 449-458, August (2018).

[3] C.H. Ting, W.H. Yeo, Y.J. King, Y.D. Chuah, J.V. Lee, \&W.B. Khaw Humanoid robot: A review of the architecture, applications and futur trend. Research Journal of Applied Sciences, Engineering/and Technology, Vol. 7(7), pp. 1364-1369, (2014).

[4] N. Sebanz, H. Bekkering, \& G. Knoblich, G. Joint aetion: bodies and) minds moving together. Trends in cognitive sciences, $10(2)$, pp. 70-76, (2006).

[5] C. Vesper, E. Abramova, J. Bütepage, F. Ciardo, B. Crossey, A. Effenberg, A., ..,\& B. Wahn. Joint action: Mental representations, shared information and general mechanisms for codrdinating with others. Front. Psychol., Vol.7, 2039, (20

[6] D. C. Dennett, "Intentional systems", J. Philos., yol. 68, no. February, 1971.

[7] D. C. Dennett, The Intentional Stance (MTT Press, 1987).

[8] N. Spatola, S. Marchesi, \& A Wykowska, A. The Instance Task: how to measure the mentalistjc bias in human-robot interaction. Front. Robot. AI - Human-Robot Lnteraction, (upder rev)

[9] E. Wiese, G. Metta \& A. Wykowska. Robots as intentional agents: using neuroscientif $\mathrm{c}$ methods to make robots appear more social. Front. Psychol., Vol.8, 1663, (2017).

[10] A. Wykowska, A. Social Robots to Test Flexibility of Human Social Cognition. International Journal of Social Robotics, pp. 1-9, (2021).

[11] J. Perez-Osorio and A. Wykowska, "Adopting the intentional stance toward natural and artificial agents", Phil. Psychol., Vol.5 (7), pp. 1$27,(2020), y$

[12] S. Marchesi, D. Ghiglino, F. Ciardo, E. Baykara, A. Wykowska, Do we adopt the intentional stance toward humanoid robots? Front. Psychol., Vol. 10, 450. (2019).

[13] G. Metta, G. Sandini, D. Vernon, L. Natale, F. Nori, "The iCub humanoid robot: an open platform for research in embodied cognition". In Proceedings of the 8th workshop on performance metrics for intelligent systems, pp. 50-56, Aug. 2009

[14] S. Marchesi, N. Spatola, J. Perez-Osorio., \& A. Wykowska. Human vs Humanoid. A behavioral investigation of the individual tendency to adopt the intentional stance. In Proceedings of the 2021 ACM/IEEE 
International Conference on Human-Robot Interaction , pp. 332-340, March 2021.

[15] F. Bossi, C. Willemse, J. Cavazza, S. Marchesi, V. Murino, A. Wykowska. The human brain reveals resting state activity patterns that are predictive of biases in attitudes toward robots, Science robotics, Vol.5 (46), (2020).

[16] B. Reeves, \& C. Nass. The media equation: How people treat computers, television, and new media like real people and places (University of Chicago Press: 1996).

[17] S. Lee, I. Lau, S. Kiesler, \& C. Chiu. Human mental models of humanoid robots. In Proceedings of the 2005 IEEE International Conference on Robotics and Automation ICRA, pp. 2767 - 2772. Barcelona, Spain, (2005).

[18] S. Marchesi, J. Perez-Osorio, D. De Tommaso, \& A. Wykowska. Don't overthink: fast decision making combined with behavior variability perceived as more human-like. In 2020 29th IEEE International Conference on Robot and Human Interactive Communication (ROMAN), pp. 54-59, Naples, Italy, (2020).

[19] S. Marchesi., F. Bossi., D. Ghiglino, D. De Tommaso, \& A. Wykowska, A. I am looking for your mind. Pupil dilation predicts individual differences in sensitivity to hints of human-likeness in robot behavior. Front. Robot. AI 8:653537 (2021)

[20] S. Mathôt,d. Schreij, J.Theeuwes, OpenSesame. An open-source, graphical experiment builder for the social sciences. Behav. Res., Vol. 44, pp.314-324 (2012)

[21] G. Metta, P. Fitzpatrick, \& L. Natale. YARP: yet another robot platform. International Journal of Advanced Robotic Systems, Vol. 3(1), p. 8, (2006).

[22] F. Ciardo, D. De Tommaso, \& A. Wykowska. Humans socially attune to their "follower" robot. In 2019 14th ACM/IEEE International Conference on Human-Robot Interaction (HRI), pp. 538-539, March, 2019.

[23] D.S. Syrdal, T. Nomura, \& K. Dautenhahn. The Frankenstein Syndrome Questionnaire-Results from a quantitative cross-cultural survey. In: Proceedings of the International Conference on Social Robotics, pp. 270-279, (2013).

[24] D.S. Syrdal., K. Dautenhahn, K.L. Kony, \& M.L. Walters. The Negative Attitudes towards Robots Scale and Reactions to Robot Behaviour in a Live Human-Robot Interaction Study. Adaptive and Emergent Behaviour and Complex Systems (2009)

[25] C.M. Carpinella, A.B. Wyman, M.A. Perez, \& S.J. Stroessner. S.J.: The Robotic Social Attributes Scale (RoSAS). In Proceedings of the ACM/IEEE International Conference on Human-Robot Interaction, pp. 254-262, (2017)

[26] JASP Team (2020). JASP (Version 0.14.1)[Computer software].

[27] M.M. de Graaf, \& S.B. Allouch. Expectation setting and personality attribution in hri. In 2014 9th ACM/IEEE International Conference on Human-Robot Interaction (HRI), pp. 144-145, March, 2014.

[28] H. Claure, \& M. Jung, M. Fairness Considerations for Enhanced Team Collaboration. In Companion of the $2021 \mathrm{ACM} / \mathrm{IEEE}$ International Conference on Human-Robot Interaction, pp. 598-600, March, 2021

[29] J.K. Burgoon. Interpersonal expectations, expectancy violations, and emotional communication. J Lang and Social Psych., Vol. 12(1-2), pp.30-48, (1993)

[30] N. A. Hinz, F. Ciardo, \& A. Wykowska. Individual differences in attitude toward robots predict behavior in human-robot interaction. In International Conference on Social Robotics, pp. 64-73, November, 2019

[31] B. Berberian, B., J.-C. Sarrazin, P. Le Blaye, \& P. Haggard. Automation technology and sense of control: a window on human agency. PLoS One, Vol. 7(3), e34075, (2012)

[32] B. Berberian, B. Somon, A. Sahaï, \& J. Gouraud. The out-of-the-loop Brain: a neuroergonomic approach of the human automation interaction. Annual Reviews in Control, Vol. 44, pp. 303-315 (2017).

[33] D.A. Norman, D. A.. The 'problem'with automation: inappropriate feedback and interaction, not 'over-automation'. Philos. Trans. Royal Soc. B, Vol. 327(1241), pp. 585-593, (1990). 